\title{
Using Time-Frequency Features to Recognize Abnormal Heart Sounds
}

\author{
Hsuan-lin Her $^{1}$, Hung-Wen Chiu ${ }^{2}$ \\ ${ }^{1}$ School of Medicine, Taipei Medical University, Taipei, Taiwan \\ ${ }^{2}$ Graduate Institute of Biomedical Informatics, Taipei Medical University, Taipei, Taiwan
}

\begin{abstract}
Disease of the heart accounts for 6\% of all death. Heart sound is a routine in physical examination clinically, and is sensitive in detecting a subset of heart diseases. In the current study, we build up a model to classify heart sounds for preclinical screening. Heart sounds are recorded under uncontrolled environment, and each sample can range from 5 to 120 seconds. This is $a$ work raised by annual PhysioNet/CinC Challenge. Because of timing and tonic natures of heart events, we used time-frequency features to classify heart sounds in this study. Firstly, each heard sound recording was segmented into cycles using Springer's improved version of Schmidt's method. Each cardiac cycle was cut into 10 partitions and data points were obtained by zero-padding in each partition. Spectral features were extracted from each partition using fast-Fourier Transform (FFT) thus a 3,500 feature matrix was created. Using filter method, 40 features were selected for the final classifier. The average feature matrix of each cycle was then applied to a classification system using 2-means clustering and artificial neural network (ANN). By clustering the unsure class was recognized. The discrimination of normal and abnormal heart sound were performed by a well-trained ANN model. The results showed that our proposed method got a performance with an accuracy $86.5 \%$, a sensitivity $84.4 \%$, a specificity $86.9 \%$. Here we show that classifying abnormal heart sound is a really difficult task due to the heterogeneity of "abnormal events" and intrasample deviation.
\end{abstract}

\section{Introduction}

A phonocardiogram is the recording of heart sounds. The sounds result from closure of cardiac valves. Two prominent heart sound, S1 and S2, representing the closure of atrioventricular valve and aortic/pulmonary valve, respectively. Splitting of heart sound or any other changes in the strength of heart sounds are indications of valvar disease, pulmonary hypertension, etc. Also, murmur is also very specific in diagnosing regurgitation. Phonocardiogram, due to its non-invasiveness, is used in screening of cardiac abnormality clinically. However, not all disease of the heart manifest itself as abnormalities in heart sounds.

Previously built models to detect abnormalities in heart sound utilize artificial neural network (ANN), support vector machine (SVM), hidden Markov model (HMM) or clustering methods. Inputs can range from wavelet features[1], time, frequency, complexity-based feature[2] and time-frequency features. Researches in digital signal processing reveal features that exceeds the capacity of traditional auscultation, allowing more sensitive detection of heart diseases. For example, Schmidt et. al propose a method of detection of coronary heart disease with PCG recordings[3], while clinically the diagnosis of coronary artery disease still depends on electrocardiogram, stress testing and other more invasive and expensive modalities[4].

Here we provide a model that can discriminate abnormal heart sounds from normal ones with machine learning. As a pre-screening tool, it needs to be robust to noise in the non-clinical environment, resulting from improper use of stethoscope. Therefore, we train our models with recordings collected in a variety of environment without specifying the precordial location.

\section{Methods}

\subsection{Subject data}

The algorithm was evaluated on data from the Computers in Cardiology 2016 Challenge, available from the Physionet website. This data consist of heart sounds source from contributors from the world, collected in either clinical or nonclinical environment, from both pathologic and healthy patients. 304 recordings are randomly selected as training data. The remainder of the recordings makes up the test data set. Both the training and test set contain about $80 \%$ normal recordings and $20 \%$ abnormal ones. 


\subsection{Segmentation of the recordings}

Figure 1 show the flow chart of data processing in this study. We first segment the heart sounds into using Springer's improved version of Schmidt's method[5,6], which uses a Hidden Markov Model (HMM) that has been trained (using database 'a' of the training set) to identify four 'states'; S1, S2, systole and diastole.

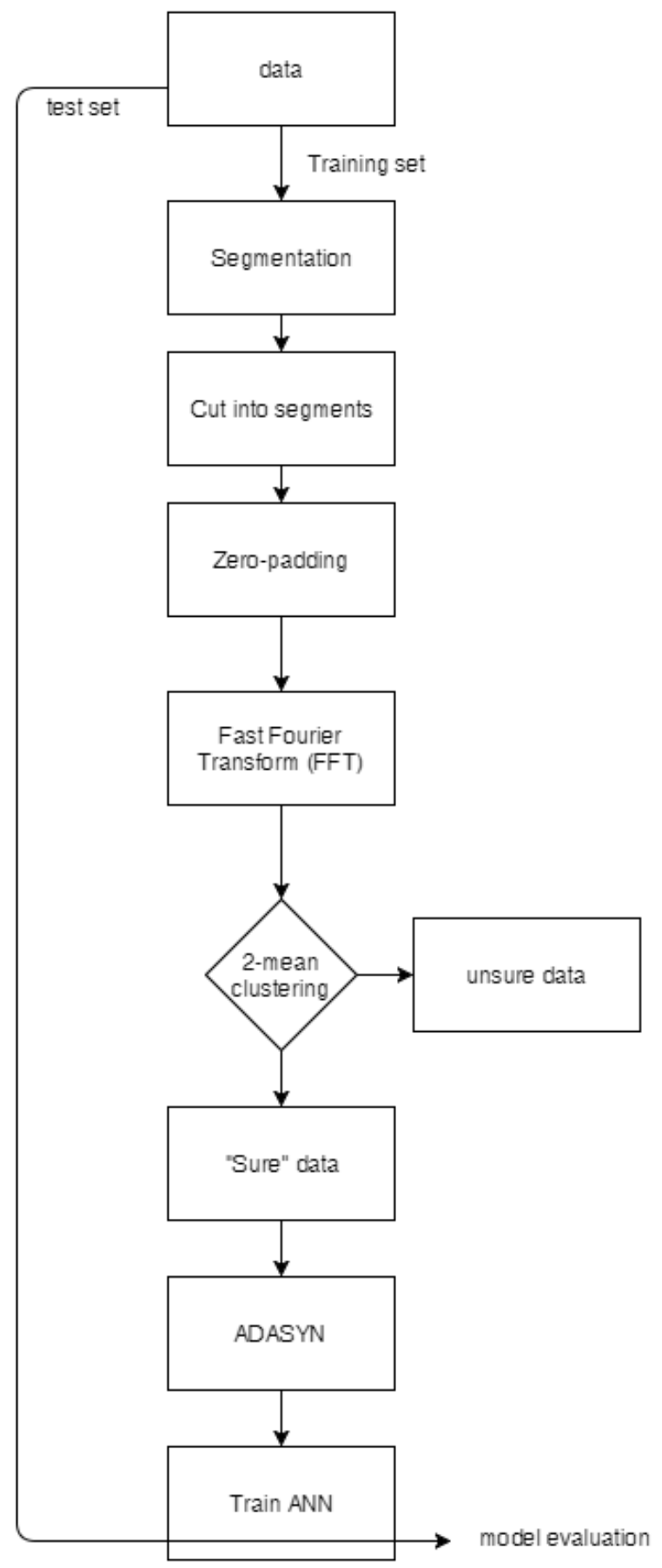

Figure 1. Flow graph of data processing. We used time- frequency features to classify heart sounds with clustering technique for unsure data and ANN as classifier.

\subsection{Feature extraction}

Each cardiac cycle was cut into 10 segments. Fast Fourier Transform (FFT) was used to characterize the time-frequency property of each segment. 250 data points were obtained after zero padding. In order not to miss the features between segments, we also extract 500 data by combining neighbouring segments. With 3,500 data points, we selected 40 features with filter methods. The average feature vector of all cycles in a single recording is used to represent each recordings.

\subsection{Identify the unsure data}

In order to detect the unsure data (e.g. non-heart sound recordings), we cluster the training data with k-mean clustering. To determine the value of $\mathrm{k}$, elbow method was used. It comes out that when $\mathrm{k}=2$, the result is optimized, suggesting there are two closed packed clusters in the data, one representing recordings that are clean and unsure ones for the other. By comparing the distance to the two centroids, the recording can be classified as 0 (unsure) or non-0.

\subsection{Dealing with imbalanced data}

Due to imbalance of data in the training set, Adaptive Synthesis(ADASYN) [7] is applied to generate synthetic data for training. Parameters of ADASYN are: beta $=1$, $k=5$, which means after ADASYN, we will have the same amount of data labelled as 1 and -1 . ADASYN further emphasize generating more synthetic data on those point that are difficult to classify, i.e. those surrounded by data points of different class. After applying ADASYN, the sensitivity improved from $58.6 \%$ to $84.4 \%$, with a little drop in accuracy (88.8\% to $86.5 \%)$

\subsection{Classifier}

The time-frequency of each recordings, a 40-element input vector (described previously in section 2.2) is fed into an ANN. A single layer feed-forward MLP with 30 hidden units was chosen. Softmax transfer function was chosen as the activation function for the output layer and tan-sigmoid function for the hidden layer. Figure 2 showed the final ANN structure.

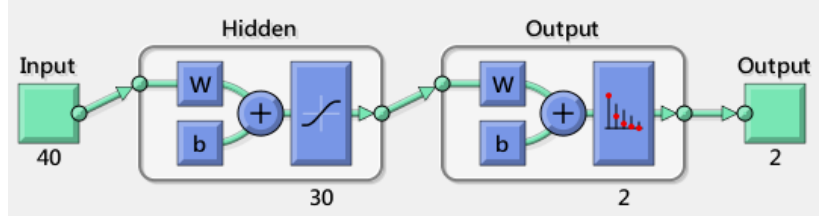

Figure 2. Artificial neural network architecture 


\section{Results}

The method proposed was tested with 304 test data. The testing results were shown in Table 1 as Confusion Matrix. The accuracy is $86.5 \%$ with a sensitivity of 84.4\% and a specificity $86.9 \%$ (We define abnormal heart sound as "positive"). The ROC curve was shown in Figure 3.

Table1. Confusion Matrix of the Classifier

\begin{tabular}{|l|l|l|l|}
\hline & $\begin{array}{l}\text { Predicted } \\
1 \\
(\text { Abnormal) }\end{array}$ & $\begin{array}{l}\text { Predicted } \\
-1 \\
(\text { Normal) }\end{array}$ & Total cases \\
\hline $\begin{array}{l}\text { Actual } \\
1\end{array}$ & 38 & 7 & $\begin{array}{l}45 \\
(84.4 \%) \\
\text { Sensitivity }\end{array}$ \\
\hline $\begin{array}{l}\text { Actual } \\
-1\end{array}$ & 34 & 225 & $\begin{array}{l}259 \\
(86.9 \%)\end{array}$ \\
& & Specificity \\
\hline & 72 & 232 & $\begin{array}{l}304 \\
(86.5 \%) \\
\text { Accuracy }\end{array}$ \\
\hline
\end{tabular}

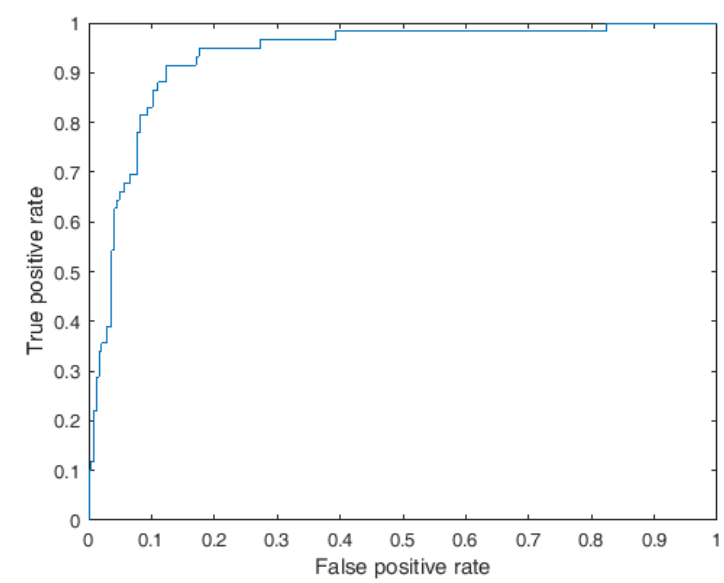

Figure 3. ROC Curve,

\section{Discussion}

For those classified as unsure, plotting reveal inability in segmentation, resulting in all-zero feature vector. The length of each cycle in the unsure group range from 1.42.2 secs, different from that of the sure group. Playing of these recording reveal poor quality in recording. For example, b0034 is intervened by talking, a0034 does not have prominent S1 S2, and e0001 is almost completely silent.

Feature selected are mostly concentrated in $16-40 \mathrm{~Hz}$, with $24 \mathrm{~Hz}$ and $32 \mathrm{~Hz}$ possessing the lowest p-value, consistent with previous research on time-frequency domain analysis on Aortic stenosis, mitral insufficiency.[8]

The misclassification of data may be due to the heterogeneity of the abnormal class. Pathologic heart sounds consists of several categories, ranging from valvar problems to congenital disorders, each possessing a very different feature. Also, how these heart sounds are labelled is also an issue. If they are labelled as abnormal merely by auscultation, only a small subset of disorders will be labelled as abnormal, since clinically most heart diseases are diagnosed with ECG. In this way, the normal class will also present heterogeneity.

Automation of auscultation is a very promising field in a future medical practitioner's point of view, because as technology evolve, machines will be able to sense more complex features than human ear. Maybe in the future, intelligent stethoscope can diagnose some severe heart disease, such as AMI, and early diagnosis and treatment can be made. To achieve this goal, more data and better models need to be generated to provide a screening method with higher sensitivity and specificity.

\section{References}

[1] Liang H, Nartimo I, editors. A feature extraction algorithm based on wavelet packet decomposition for heart sound signals. Time-Frequency and Time-Scale Analysis, 1998 Proceedings of the IEEE-SP International Symposium on; 1998: IEEE

[2] Schmidt SE, Græbe M, Toft E, Struijk JJ. No evidence of nonlinear or chaotic behavior of cardiovascular murmurs. Biomedical Signal Processing and Control. 2011;6(2):15763.

[3] Semmlow J, Rahalkar K. Acoustic detection of coronary artery disease. Annu Rev Biomed Eng. 2007;9:449-69.

[4] Cassar A, Holmes DR, Rihal CS, Gersh BJ. Chronic Coronary Artery Disease: Diagnosis and Management. Mayo Clin Proc. 2009;84(12):1130-46.

[5] Schmidt S, Holst-Hansen C, Graff C, Toft E, Struijk JJ. Segmentation of heart sound recordings by a durationdependent hidden Markov model. Physiological Measurement. 2010;31(4):513.

[6] Springer DB, Tarassenko L, Clifford GD. Logistic regression-hsmm-based heart sound segmentation. IEEE Transactions on Biomedical Engineering. 2016;63(4):82232.

[7] Haibo H, Yang B, Garcia EA, Shutao L, editors. ADASYN: Adaptive synthetic sampling approach for imbalanced learning. 2008 IEEE International Joint Conference on Neural Networks (IEEE World Congress on Computational Intelligence); 2008 1-8 June 2008.

[8] Ahlström C. Processing of the Phonocardiographic Signal: methods for the intelligent stethoscope. 2006.

Hung-Wen Chiu

No. 250, Wuxingst, Xing-yi District, Taipei, Taiwan.

hwchiu@tmu.edu.tw 
